\title{
INSTABILITIES AND SMALL-SIGNAL RESPONSE OF DOUBLE INJECTION STRUCTURES WITH DEEP TRAPS
}

\author{
W. H. WEBER \\ Scientific Research Staff, Ford Motor Company, Dearborn, Michigan 48121, U.S.A. \\ and \\ G. W. FORD \\ Department of Physics, University of Michigan, Ann Arbor, Michigan 48104, U.S.A.
}

(Received 13 April 1972; in revised form 30 May 1972)

\begin{abstract}
The small-signal response is discussed for double injection structures heavily doped with deep recombination centers. The discussion is based on an exact small-signal linearization of the nonlinear equations describing the steady state. Diffusion is neglected, but space charge, thermal emission, and position- and injection-level-dependent lifetimes are included. The problem is reduced to finding the solution of a single second order linear differential equation, which is valid at any injection level. Approximate general solutions are derived for high and low frequencies. Numerical solutions are given for Au-doped silicon $p-i-n$ devices operating in the low injection square-law regime. Previously a much simpler model was used to calculate the small-signal response in this regime, and that model was successful in explaining the spontaneous oscillations observed there. It is demonstrated that the numerical solutions to the exact equation give results very similar to the previous model. A physical description of the space-charge recombination oscillations is given. This description suggests that the space charge on the traps and its phase shift with respect to the free carriers are the important factors giving rise to the oscillatory instabilities.
\end{abstract}

Résumé-La réponse à faible signal pour des structures à double injection fortement additionnées de centres profonds de recombinaison est discutée. La discussion s'appuie sur une linéarisation exacte à faible signal des équations nonlinéaires décrivant l'état stationnaire. La diffusion est négligée mais la charge d'espace, l'émission thermique et les périodes de vie dépendant de la position et du niveau d'injection sont mises en ligne de compte. Le problème se ramène à trouver la résolution d'une seule équation différentielle linéaire de deuxième ordre, qui est valable pour tout niveau d'injection. Des résolutions générales d'approximation sont déduites pour les hautes et les basses fréquences. On offre des résolutions numériques pour des des dispositifs $p-i-n$ de silicium additionés d'Au fonctionnant dans des régimes de loi carrée d'injection basse. Un modèle beaucoup moins complexe a été employé antérieurement pour le calcul de la réponse à faible signal dans ce régime, et ce modèle pouvait expliquer avec succès les oscillations spontanées y observées. On démontre que les résolutions numériques de l'équation axacte offrent des résultats très semblables à ceux du modèle antéieur. Une description physique des oscillations de la recombinaison de charge d'espace est donnée. Cette description suggère que la charge d'espace sur les trappes et son déphasage à l'égard des porteurs de charges libres sont les facteurs essentiels dans l'origine des instabilités oscillatoires.

Zusammenfassung - Das Wechselstromverhalten von Doppelinjektionstrukturen, stark dotiert mit tiefliegenden Rekombinationszentren, wird diskutiert. Die Diskussion basiert auf eine, für kleine Signale, cxakte Lincarisation der nichtlinearen Gleichungen welche den Dauerzustand beschreiben. Diffusion wird vernachlässigt, jedoch Raumladung, thermische Emission, und position-und injekionsniveauabhängige Lebensdauer werden berïcksichtigt. Das Problem wird darauf reduziert, die Lösung für eine lineare Differentialgleichung zweiter Ordnung zu finden die für jedes Injektionsniveau gültig ist. Angenäherte generelle Lösungen für hohe und niedrige Frequenzen werden abgeleitet. Numerische Lösungen für Au-dotierte Silizium $p-i-n$ Dioden die im Bereich der bei niedriger Injektion quadratischen Abhängigkeit arbeiten werden gegeben. Ein wesentlich einfacheres Modell wurde früher benutzt um die Wechselstromverhalten in diesem Bereich zu berechnen. Dieses Modell erklärte erfolgreich die dort beobachteten spontanen Oszillationen. Es wird gezeigt, dass die numeris- 
chen Lösungen der exakten Gleichungen Resultate ergeben die sehr ähnlich zu denen des vorherigen Modells sind. Eine physikalische Reschreibung der Raumladungs-Rekombinationsoszillationen wird gegeben. Diese Beschreibung deutet an, das die Raumladung der Rekombinationzentren und seine Phasenverschiebung gegenüber den freien Ladungsträgern die primäen Faktoren sind die zu Oszillationsinstabilitäten führen.

\section{NOTATION}

$A$ d.c. quantity proportional to the ratio of the space charge to the recombination rate

$B$ d.c. quantity inversely proportional to the recombination rate

$a, b$ small-signal expansion parameters for the charge density

$\alpha, \beta$ small-signal expansion parameters for the recombination rates

$c_{n}{ }^{+} 6.3 \times 10^{-8} \mathrm{~cm}^{3} / \mathrm{sec}$, capture coefficient for electrons on positive gold centers [36]

('n $1.65 \times 10^{-9} \mathrm{~cm}^{3} / \mathrm{sec}$, capture coefficient for electrons on neutral gold centers [36]

$c_{p}{ }^{-} 11.5 \times 10^{-8} \mathrm{~cm}^{3} / \mathrm{sec}$, capture coefficient for holes on negative gold centers [36]

$c_{p} 2.4 \times 10^{-k} \mathrm{~cm}^{3} / \mathrm{sec}$, capture coefficient for holes on neutral gold centers [36]

$e_{p} 500 \mathrm{sec}{ }^{1}$, thermal emission rate for holes from neutral gold centers

$e_{p}{ }^{+} 7.7 \times 10^{5} \mathrm{sec}^{-1}$, thermal emission rate for holes from positive gold centers

$e_{n} \quad 0.38 \mathrm{sec}^{-1}$, thermal emission rate for electrons from neutral gold centers

$e_{n}{ }^{-} 74 \mathrm{sec}^{-1}$, thermal emission rate for electrons from negative gold centers

e $1.6 \times 10^{-19} \mathrm{C}$, electronic charge

$E_{0}, E$ d.c. and time dependent electric fields, respectively

$€$ dimensionless d.c. electric field variable

$\epsilon 1.04 \times 10^{-12} \mathrm{~F} / \mathrm{cm}$, dielectric constant of silicon

$F E / E_{0}$, dimensionless time dependent field

$j_{0}, j$ d.c. and time dependent current densities, respectively

$j_{n}, j_{j} \quad$ electron and hole time dependent current densities, respectively

$J j_{0} / e N_{n}{ }^{2}\left(c_{n}+c_{p}\right) L$, dimensionless current density

$L \quad$ length of the $i$-region, typically $100 \mu \mathrm{m}$

$M$ large dimensionless constant, $-2 \times 10^{\mathrm{3}}$ for gold-doped silicon

$n, p$ time dependent electron and hole number densities, respectively

$n_{0}, p_{0} \quad$ d.c. electron and hole number densities, respectively

$N, P$ time dependent number densities of the negative and positive gold centers, respectively

$N_{0}, P_{0}$ d.c. number densities of negative and positive gold centers, respectively

$N_{R}$ total number density of gold recombination centers

$n_{i} \quad 1.4 \times 10^{10} \mathrm{~cm}^{-3}$, carrier density in intrinsic silicon at $300^{\circ} \mathrm{K}$

$s \quad$ Laplace transformed time variable

$\tau_{n}, \tau_{n}$ electron and hole lifetimes, respectively.
(Their values in the low injection square-law regime with $N_{k}=10^{16} \mathrm{~cm}^{-3}$ are $18.2 \mathrm{nsec}$ and $3.53 \mathrm{nsec})$

$\tau_{i^{\prime}} \epsilon\left(\mu_{n}+\mu_{p}\right) / e \mu_{n} \mu_{p} N_{n}$

$\tau_{\epsilon} \quad \epsilon\left(\mu_{n} \tau_{u}+\mu_{p} \tau_{p}\right) / e \mu_{n} \mu_{n}\left(p_{0} \tau_{n}-n_{n} \tau_{n}\right)$

$\tau_{1} \quad \tau_{n} \tau_{\mu}\left(\mu_{n}+\mu_{1}\right) /\left(\mu_{n} \tau_{n}+\mu_{,} \tau_{\mu}\right)$

$\mu_{\mu}, \quad 480 \mathrm{~cm}^{2} / \mathrm{V}-\mathrm{sec}$, hole mobility at $300^{\circ} \mathrm{K}$

$\mu_{\prime \prime} \quad 1350 \mathrm{~cm}^{2} / \mathrm{V}-\mathrm{sec}$, electron mobility at $300^{\circ} \mathrm{K}$

$V_{0}$ d.c. voltage drop across the $i$-region

$U, V, W, T$ coefficients in the master equation for the time dependent field

$x$ position coordinate

$z$ dimensionless quantity which effectively replaces $x$ as a position variable

$z_{i 1}, z_{i}$ values of $z$ at the anode and cathode, respectively

$Z$ small-signal impedance function

\section{INTRODUCTION}

INSTABILITIES in double injection structures were first observed by Holonyak and Bevacqua in 1963 [1]. These structures consist of a long high resistivity $i$-region with a hole injecting contact on one side (the anode) and an electron injecting contact on the other (the cathode). The $i$-region is a semiconductor heavily doped with a deep recombination center. When such devices are operated under forward bias, spontaneous oscillations in the current and voltage occur. Although at high voltages such structures exhibit a negative resistance in the d.c. characteristic, the oscillations generally occur at lower voltages in a positive resistance region. The original observations of Holonyak and Bevacqua were on $\mathrm{Au}-, \mathrm{Co}-$, and $\mathrm{Zn}$-doped Si and GaAs with an unknown impurity. Since then similar instahilities have been observed in $\mathrm{Si}, \mathrm{Ge}$, and GaAs with a wide variety of impurities providing the recombination centers [2-7]. These oscillations have been found even when the recombination centers are formed by radiation damage [8]. It appears, therefore, that the instability is a general phenomenon not related to details of the transport processes or of the recombination mechanisms in the semiconductor. The presence of both electrons and holes and of relatively large impurity densities seem to be essential, however.

These instabilities are remarkable in that they are not associated with an instability or negative 
resistance in the d.c. characteristic. In this sense they are reminiscent of the instabilities associated with the onset of turbulence in fluid flow [9]. Most other known oscillatory effects in semiconductors which do not involve a magnetic field arise from a d.c. instability of some sort, e.g., the Gunn effect, impact ionization, Zener breakdown, or field dependent trapping; and in order to obtain narrow band oscillations from instabilities of this type an external resonant circuit is generally required. In contrast double injection devices exhibit very narrow band oscillations $\left(\Delta f / f<10^{-3}\right)$ without an external resonant circuit.

The first attempt to explain the origin of these oscillations was by Konstantinov and Perel $[10,11]$ who discussed a model in which they included the effects of diffusion but neglected space charge. They found instabilities referred to as 'recombination waves' in an infinite medium, but they incorrectly applied the boundary conditions for a finite length device. Antognetti et al. $[12,13]$ recently extended their model and correctly included the influence of the boundaries on the threshold conditions. From a theoretical point of view we have two criticisms of the recombination wave model in its present form. The first is that all the d.c. quantities and their spatial dependences have been approximated in a very crude fashion, and no attempt has been made to demonstrate the validity of these approximations. The second, which is much more severe, is that the spacecharge terms which have been neglected in the small-signal equations are, in fact, much larger for a typical device than the diffusion terms which have been kept. We will establish this point later using a simple dimensional analysis. In addition to the above theoretical shortcomings of the model, it also does not appear to satisfactorily explain the experimental results. Holonyak and co-workers $[2,3,14]$ have established that for a given material the voltage at which oscillations first occur is proportional to the device length squared, while the frequency at threshold has no length dependence. The threshold frequency and voltage can also have strong temperature dependences, and the oscillation frequency changes as the voltage is increased above threshold. Of these results, the independence of the threshold frequency on the device length is apparently the only one given by the recombination wave model.

There have been several other more qualitative attempts to explain the observed oscillations, but none have led to meaningful predictions. The most prominent of these is a space-charge relaxation mechanism predicting oscillations with a period given by the electron lifetime [14,15]. Although this model is consistent with the dependence of the oscillation frequency on the gold density observed by Moore [14] in Au-doped silicon $p-i-n$ diodes, it fails to explain how such a relaxation mechanism can give narrow band sinusoidal oscillations. In addition we have observed in Au-doped silicon diodes that the oscillation period can be much different from the electron lifetime even though each of these quantities scales roughly in the same way with the impurity density.

The small-signal response of double injection structures has been discussed by Baron, Marsh, and Mayer[16] and others[17-21] for devices biased in the high injection regimes, i.e., the regimes above the negative resistance in heavily doped semiconductors. The response in the low injection regime, however, has been treated only under the limited assumption of quasineutrality [22]. None of these treatments has predicted snontaneous oscillations.

In this paper we discuss the small-signal response for long double injection structures operating in the low injection square-law regime, which occurs below the negative resistance region. We include space-charge effects, but neglect diffusion. We will have in mind the particular example of high purity silicon doped with gold, and specific results will be given only for that case. The method of discussion, however, is general. In an earlier paper we gave an approximate discussion of this problem in which we neglected the spatial variation of the d.c. variables [23]. There we showed that spontaneous oscillations occur whose threshold properties are consistent with experimental observations. Here we will give a more rigorous treatment of the problem, using the correct d.c. solution. The results we obtain are very similar.

In Section 2 we formulate the problem and reduce it to the problem of solving a single second order differential equation for the time dependent electric field. This equation is the exact smallsignal linearization of the nonlinear steady-state problem; it involves no additional approximations. In Section 3 we construct several approximate solutions and discuss the resulting impedance function for each of these. In Section 4 we describe 
a method for finding exact numerical solutions. Computer results are given for Au-doped silicon $p-i-n$ diodes operating in the low-injection square-law regime. A physical picture of the spacecharge recombination waves is also presented in Section 4.

\section{FORMULATION OF THE PROBLEM}

\subsection{Steady state equations}

Our starting point is a brief review of the steadystate solution for long double injection structures (diffusion is neglected) based on the original model of Lampert and Rose [24] and Lampert [25] and extended to the case of deep traps by Ashley [26] and others[27-31]. Our notation will follow closely that in Ref. [28].

The equations describing the d.c. solution are the electron and hole continuity equations and Poisson's equation:

$$
\begin{array}{r}
\frac{1}{e} \frac{\mathrm{d}}{\mathrm{d} x} j_{n, 0}(x)= \\
\frac{\epsilon}{e} \frac{\mathrm{d}}{\mathrm{d} x} j_{p, 0}(x)=r_{0}(x), \\
\frac{\mathrm{d} E_{0}(x)}{\mathrm{d} x}=p_{0}(x)-n_{0}(x)+P_{0}(x) \\
-N_{0}(x)+N_{d^{+}}-N_{0}{ }^{-},
\end{array}
$$

where $e$ is the electronic charge; $j_{n, 0}$ and $j_{p, 0}$ are the electron and hole current densities; $r_{0}$ is the net recombination rate; $E_{0}$ is the electric field strength; $\epsilon$ is the dielectric constant; $n_{0}$ and $p_{0}$ are the electron and hole number densities; $N_{0}$ and $P_{0}$ are the trapped negative and positive charge number densities; $N_{d}^{+}$and $N_{a}^{-}$are the number densities of the shallow ionized donors and acceptors; and $x$ is the position variable measured from the anode $(x=0)$. These equations are to be solved subject to the boundary conditions:

$$
E_{0}(0)=E_{0}(L)=0,
$$

where $L$ is the device length.

We first introduce a dimensionless d.c. electric field $\mathscr{E}$ defined by

$$
\mathscr{E}(x)=\frac{e \mu_{n} \mu_{p} N_{R}}{j_{0}\left(\mu_{n}+\mu_{p}\right)} E_{0}(x),
$$

where $\mu_{n}$ and $\mu_{p}$ are the electron and hole mobilities, $N_{R}$ is the deep impurity density, and $j_{0}$ is the d.c. current density. Next we introduce a dimen- sionless quantity $z$, which is a measure of the relative fraction of the current carried by each carrier type:

$$
z=\frac{j_{n, 0}-j_{p, 0}}{j_{0}}=\frac{\mu_{n} n_{0}-\mu_{p} p_{0}}{\mu_{n} n_{0}+\mu_{p} p_{0}}
$$

Since $z$ increases monotonically from the anode to the cathode, it effectively replaces $x$ as the position variable in the problem. The total current

$$
j_{0}=e\left(\mu_{n} n_{0}+\mu_{p} p_{0}\right) E_{0}
$$

is constant, thus we can express the carrier densities in terms of $z$ and $\mathscr{E}$ :

$$
n_{0}=\frac{\mu_{p} N_{k}}{\mu_{n}+\mu_{p}} \frac{1+z}{2 \mathscr{E}}, \quad p_{0}=\frac{\mu_{n} N_{R}}{\mu_{n}+\mu_{p}} \frac{1-z}{2 \mathscr{E}} .
$$

From the continuity equations (1) it follows that

$$
\frac{\mathrm{d} z}{\mathrm{~d} x}=\frac{2 e}{j_{0}} r_{1)} .
$$

For a particular set of trap levels $r_{0}$ is determined as a function of $n_{0}$ and $p_{0}$ from the ShockleyRead-Hall $[32,33]$ formalism for the recombination kinetics. Using (6) thus allows one to express $r_{0}$ as a function of $z$ and $\mathscr{E}$.

Now we divide (2) by (7), and the result is a first order nonlinear differential equation for $\mathscr{E}(z)$ :

$\frac{\mathrm{d} \mathscr{E}}{\mathrm{d} z}=\frac{e \mu_{n} \mu_{p} N_{R}}{2 \epsilon\left(\mu_{n}+\mu_{p}\right)} \frac{p_{0}-n_{0}+P_{0}-V_{0}+N_{d}^{+}-N_{a}}{r_{0}(z, \mathscr{E})}$.

Note that the right side of (8) can be expressed as a function of $z$ and $\mathscr{E}$ by using the recombination kinetics and (6).

To complete the d.c. solution one must determine solutions $\mathscr{E}(z)$ to equation (8). In practice this must be done numerically as was done in Ref. [28]. Each such solution will vanish at some pair of points $z_{0}$ and $z_{L}$, the values of $z$ at the anode and cathode, respectively; and the corresponding current and voltage for each are determined by evaluating the integrals:

$$
j_{0}=2 e L\left\{\int_{z v}^{z_{L}} \mathrm{~d} z r_{0}(z, \mathscr{C})^{-1}\right\}^{-1},
$$




$$
V_{0}=\frac{j_{0}}{2 e} \int_{z 0}^{z_{L}} \mathrm{~d} z \frac{E_{0}(z)}{r_{0}(z, \mathscr{E})}
$$

In the discussion of the d.c. solution it was convenient to express the right hand sides of equations (7) and (8) in terms of two dimensionless functions $B(z, \mathscr{E})$ and $A(z, \mathscr{E})$, defined by the relations:

$$
\begin{aligned}
& \frac{\mathrm{d} z}{\mathrm{~d} x}=\frac{1}{J L B(z, \mathscr{E})}, \\
& \frac{\mathrm{d} \mathscr{E}}{\mathrm{d} z}=M A(z, \mathscr{E}),
\end{aligned}
$$

where $J$ is a dimensionless current density, and $M$ is a large dimensionless constant. For the case of Au-doped silicon we have taken

$J=\frac{j_{0}}{e N_{R}^{2}\left(c_{n}+c_{p}\right) L}, \quad M=\frac{e \mu_{n} \mu_{p}}{\epsilon\left(\mu_{n}+\mu_{p}\right)\left(c_{n}+c_{p}\right)}$,

where $c_{n}$ and $c_{p}$ are the capture coefficients for electrons and holes, respectively, onto neutral gold atoms. For an arbitrary set of trap levels any representative choice of capture coefficients could be used in place of $c_{n}+c_{p}$ in the definitions (13). Note that for a given d.c. solution $\mathscr{E}(z)$ is determined, and thus $B$ and $A$ are functions of $z$ alone.

\subsection{Small-signal equations}

To discuss the small-signal problem we linearize about the d.c. solution. That is, we take the time dependent quantities to be equal to their d.c. values plus a small part varying in time as exp $(s t)$, e.g., $n(x, t)=n_{0}(x)+n(x) \exp (s t)$. The smallsignal analogues of equations (1) and (2) become

$$
\begin{gathered}
\frac{1}{e} \frac{\mathrm{d} j_{n}}{\mathrm{~d} x}=s n+r_{n}, \\
-\frac{1}{e} \frac{\mathrm{d} j_{p}}{\mathrm{~d} x}=s p+r_{p}, \\
\frac{\epsilon}{e} \frac{\mathrm{d} E}{\mathrm{~d} x}=p-n+P-N,
\end{gathered}
$$

where $r_{n}$ and $r_{p}$ are the small-signal electron and hole recombination rates. Since the time rate of change of the trapped charge must equal the difference between the hole and electron re- combination rates, we have the further relation:

$$
s(P-N)=r_{p}-r_{n} .
$$

The total time dependent current

$$
j=j_{n}+j_{p}+s \epsilon E
$$

is constant as can be seen by adding the time derivative of equation (16) to the difference of equations (14) and (15) and then using (17).

From the recombination kinetics the quantities $P, N, r_{n}$, and $r_{p}$ can all be expanded as linear combinations of $n$ and $p$. We can therefore write

$$
\begin{gathered}
n-p+N-P=a n-b p, \\
s(n+p)+r_{n}+r_{p}=\left(c_{n}+c_{p}\right) N_{R}(\alpha n+\beta p),
\end{gathered}
$$

where $a, b, \alpha$, and $\beta$ are dimensionless expansion parameters. Explicit expressions for these parameters, which are rather cumbersome functions of the d.c. solution, are given in the Appendix. The time varying parts of the electron and hole current densities are

$j_{n}=e \mu_{n}\left(n_{0} E+E_{0} n\right), \quad j_{p}=e \mu_{p}\left(p_{0} E+E_{0} p\right)$.

Using equation (16) with (18), (19), and (21), we can express the carrier densities in terms of the time-dependent field:

$$
\begin{aligned}
& p=\frac{\mu_{n} \epsilon E_{0}(\mathrm{~d} E / \mathrm{d} x)+a\left(j-j_{0} E / E_{0}-s \epsilon E\right)}{e E_{0}\left(\mu_{n} b+\mu_{p} a\right)} \\
& n=\frac{-\mu_{p} \epsilon E_{0}(\mathrm{~d} E / \mathrm{d} x)+b\left(j-j_{0} E / E_{0}-s \epsilon E\right)}{e E_{0}\left(\mu_{n} b+\mu_{p} a\right)} .
\end{aligned}
$$

We next form the sum of equations (14) and (15):

$$
\frac{1}{e} \frac{\mathrm{d}}{\mathrm{d} x}\left(j_{n}-j_{p}\right)=s(n+p)+r_{n}+r_{p} .
$$

For the right side of equation (23) we substitute (20) and (22), while for the left side we use (21) and (22). The result is a single differential equation for $E(x)$. This equation can be simplified by introducing a dimensionless time dependent field

$$
F=E / E_{0}
$$

and replacing $(\mathrm{d} / \mathrm{d} x)$ by $(J L B)^{-1}(\mathrm{~d} / \mathrm{d} z)$ as in the d.c. 
solution. The motivation for this last step is the fact that many of the expressions relating to the steady-state solution can be evaluated easily as functions of $z$, while their dependence on $x$ can only be determined by integrating (11). We obtain for the final result:

$$
\begin{aligned}
& \frac{\mathrm{d}}{\mathrm{d} z}\left\{z F-\frac{2\left(\mu_{n}+\mu_{p}\right)}{\mu_{n} b+\mu_{p} a} \frac{\mathscr{E}}{M B} \frac{\mathrm{d}}{\mathrm{d} z} \mathcal{E} F+\frac{\mu_{n} b-\mu_{p} a}{\mu_{n} b+\mu_{p} a}\right. \\
& \left.\times\left(\frac{j}{j_{n}}-F-s \tau_{y} \mathscr{E} F\right)\right\}=\frac{\mu_{n} \beta-\mu_{\nu} \alpha}{\mu_{n} b+\mu_{\mu} a} \frac{1}{M} \frac{\mathrm{d}}{\mathrm{d} z} F \\
& +\frac{\mu_{n} \mu_{p}}{\mu_{n}+\mu_{p}} \frac{a \beta+b \alpha}{\mu_{n} h+\mu_{n, t} r} \frac{B}{\mathscr{C}}\left(\frac{j}{j_{0}}-F-s \tau_{V} \mathscr{E} F\right),
\end{aligned}
$$

where

$$
\tau_{N}=\frac{\epsilon\left(\mu_{n}+\mu_{p}\right)}{e \mu_{n} \mu_{p} N_{k}}
$$

and we have used equations (4), (5), and (13). This is an inhomogeneous, second order, linear equation for $F(z)$. It must be solved subject to the boundary conditions that the time dependent electric field vanish at the ends of the device. Since $E_{11}(z)$ satisfies these boundary conditions, this requires only that $F(z)$ be regular at $z_{i}$ and $z_{l}$.

Equation (24) is the master equation for determining the small-signal response of a double injection diode in any regime of operation. For a particular value of $s$ we must determine a solution $F(z)$ to (24). With this solution we evaluate the small-signal impedance given by

$$
Z(s)=\frac{1}{j} \int_{0}^{L} \mathrm{~d} x E(x)=\frac{J L}{j} \int_{z 0}^{z_{L}} \mathrm{~d} z B(z) E_{0}(z) F(z),
$$

or, using the results from the steady state solution,

$$
Z(s)=R_{0} \frac{j_{0}}{j} \frac{\int_{z \Downarrow}^{z_{l}} \mathrm{~d} z B \mathscr{E} F}{\int_{z_{0}}^{z_{l}} \mathrm{~d} z B \mathscr{E}}
$$

where $R_{0} \equiv V_{0} / j_{0}$ is the d.c. resistance, Knowing this impedance as a function of $s$ we can apply Nyquist's criterion to answer the question of stability: Zeros or poles of $Z(s)$ which occur in the right half $s$ plane correspond to spontaneous oscillations of current at constant voltage or voltage at constant current, respectively.

It is a general property of the system of equations describing the steady state that if the device length is changed the current scales as $L$ and the voltage as $L^{2}$. For a given $V_{0} / L^{2}$, the solutions for any device length will have exactly the same spatial variations. This result also carries over to the small-signal problem. This means that if we find poles or zeros in $Z(s)$ which move with voltage, crossing into the right half $s$ plane at some threshold values, then the threshold voltage will scale as $L^{2}$ and the threshold frequency will be independent of $L$.

The primary simplification we have made in our discussion is the neglect of diffusion effects. This approach is motivated by the results from the steady state in which diffusion was shown to be unimportant for devices long compared with the ambipolar diffusion length[21]. The alternative treatment of this problem, used in the recombination wave model, neglects space-charge effects and includes diffusion in the small-signal equations. Necessary conditions for the validity of the two methods can be derived by a simple dimensional analysis. We introduce the diffusion terms into the small-signal currents $(21)$, replace all derivatives with respect to $x$ by $L^{-1}$, and insert average values for all steady state variables. The resulting master equation will have three kinds of terms: those involving diffusion, those involving space charge, and those involving neither, which we call the drift terms. If we normalize all terms with respect to one of the drift terms, then we can identify dimensionless quantities characterizing the relative magnitudes of those remaining. For the diffusion terms the appropriate quantity is $\left(k T / e E_{0} L\right)$, and for a typical device (Au-doped $\mathrm{Si}$, $N_{n}=10^{16} \mathrm{~cm}^{-3}, L=100 \mu \mathrm{m}, T=300^{\circ} \mathrm{K}, V_{n}=20 \mathrm{~V}$ ) its magnitude is roughly $10^{-3}$. The neglect of diffusion thus appears to be a reasonable approximation. The corresponding quantity for the important space-charge terms is $\left(\epsilon \mu_{n} E_{0}{ }^{2} /\left(i j_{0} L\right)\right.$, which is simply the ratio of the first to the third terms in the numerator of equation (22). For the same typical device[28] this quantity is of order unity. The conclusion is that space charge must be included in any accurate treatment of these devices. Diffusion may indeed be more important than the above argument suggests, but its effect can only be determined by a detailed treatment which properly takes the contact regions into account. 


\section{APPROXIMATE SOLUTIONS}

\subsection{General remarks}

Before attempting to obtain any approximate solutions we want to first discuss the general properties of equation (24). This requires a description of its singular points, and for this purpose we rewrite it in the more conventional form:

$$
U(z) \frac{\mathrm{d}^{2} F}{\mathrm{~d} z^{2}}+V(z) \frac{\mathrm{d} F}{\mathrm{~d} z}+W(z) F=\frac{j}{j_{0}} T(z),
$$

where

$$
\begin{gathered}
U(z)=-\frac{2\left(\mu_{n}+\mu_{p}\right)}{\mu_{n} b+\mu_{p} a} \frac{\mathscr{E}^{2}}{M B}, \\
V(z)=z-2 \frac{\mu_{n}+\mu_{p}}{M} \frac{\mathrm{d}}{\mathrm{d} z}\left\{\frac{\mathscr{E}^{2} B^{-1}}{\mu_{n} b+\mu_{p} a}\right\} \\
-2 \frac{\mu_{n}+\mu_{p}}{\mu_{n} b+\mu_{p} a} \frac{\mathscr{E} A}{B}-\frac{\mu_{n} b-\mu_{p} a}{\mu_{n} b+\mu_{p} a}\left(1+s \tau_{N} \mathscr{E}\right) \\
-\frac{\mu_{n} \beta-\mu_{p} \alpha}{\mu_{n} b+\mu_{p} a} \frac{\mathscr{E}}{M}, \\
W(z)=1-2\left(\mu_{n}+\mu_{p}\right) \frac{\mathrm{d}}{\mathrm{d} z}\left\{\frac{\mathscr{E} A B^{-1}}{\mu_{n} b+\mu_{p} a}\right\} \\
+\left(1+s \tau_{N} \mathscr{E}\right) T(z)-\frac{\mu_{n} b-\mu_{p} a}{\mu_{n} b+\mu_{p} a} s \tau_{N} M A \\
-\frac{\mu_{n} \beta-\mu_{p} \alpha}{\mu_{n} b+\mu_{p} a} A,
\end{gathered}
$$$$
T(z)=\frac{\mu_{n} \mu_{p}}{\mu_{n}+\mu_{p}} \frac{a \beta+b \alpha}{\mu_{n} b+\mu_{p} a} \frac{B}{\mathscr{E}}-\frac{\mathrm{d}}{\mathrm{d} z}\left\{\frac{\mu_{n} b-\mu_{p} a}{\mu_{n} b+\mu_{p} a}\right\} .
$$

The singular points of equation (28) are those points at which $U(z)$ is zero.

At the ends of the device the field vanishes, and the carrier densities, as well as their accompanying space charge, diverge as $\mathscr{E}^{-1}$ in order to maintain constant current. The trapped charge densities and the carrier lifetimes will approach constants determined by the ratio $\left(p_{0} / n_{0}\right)$. The recombination rate $r_{0}$ becomes $n_{0} / \tau_{n}\left(=p_{0} / \tau_{p}\right)$ and will also diverge as $\mathscr{E}^{-1}$. Thus both the numerator and denominator in equation (8) will diverge as $\mathscr{E}^{-1}$ at the contacts. This means that $A(z, \mathscr{E})$ will become a function of $z$ alone at the ends of the device, and we can write

$$
\begin{aligned}
& \mathscr{E}(z) \cong \operatorname{MA}\left(z_{0}\right)\left(z-z_{0}\right), \quad z \rightarrow z_{0}, \\
& \mathscr{E}(z) \cong \operatorname{MA}\left(z_{L}\right)\left(z-z_{L}\right), \quad z \rightarrow z_{L},
\end{aligned}
$$

where $A\left(z_{0}\right)>0$ and $A\left(z_{l}\right)<0$. At the same time $B$ will become proportional to $\mathscr{E}(z)$, and $a$ and $b$ will become unity at the contacts. The result is that $U(z)$ will tend to zero at $z_{i}$ and $z_{L}$ as $\mathscr{E}(z)$ does. As long as $s$ is not on the negative real axis, these are the only points at which $U(z)$ vanishes. On the other hand, $V(z)$ and $W(z)$ remain finite (nonzero) at the contacts. Thus, the points $z_{0}$ and $z_{L}$ are regular singular points of the differential equation (28)[34]. At a singular point one of the two solutions of the homogeneous equation will in general be irregular. Hence, when there are two singular points, the solution regular at one will generally be irregular at the other, and there are no solutions to the homogeneous equation regular at both singular points. This implies a unique solution to the inhomogeneous equation (28).

Since the expressions for $U, V, W$, and $T$ are so complicated, it is perhaps more instructive to give actual numerical results for a particular set of parameters rather than to attempt a general discussion. In Fig. 1 we show computed curves for these coefficients at two different values of $s$ for the case of a Au-doped silicon $p-i-n$ diode with $N_{R}=10^{16} \mathrm{~cm}^{-3}, L=100 \mu \mathrm{m}$, and $V_{0}=15.7 \mathrm{~V}$. The most prominent features in these curves are the very sharp structures which occur near $z=0.5$ and $z=0.87$. These structures correspond physically to the space-charge regions near the anode and cathode, respectively. The structure near the anode occurs throughout the low injection square-law regime, moving to larger values of $z$ as the voltage is increased. The structure near the cathode occurs in both square-law regimes and moves to smaller values of $z$ as the high injection cube-law region is approached. Another important result indicated in Fig. 1 is that over most of the range of $z U(z)$ and $V(z)$ are quite small compared with $W(z)$ and $T(z)$. This fact will allow us to obtain approximate solutions for $F(z)$ at very low and very high frequencies, as will be discussed below.

\subsection{Large s behavior}

For very large values of $s$ we first note that $a$ and $b$ become unity, while $\alpha$ and $\beta$ become propor- 
tional to $s$. These results follow by inspection of equations (A3) and (A7) in the Appendix. This means that $T(z)$ will increase as $s$, while $W(z)$ increases as $s^{2}$. Now, keeping only the highest order terms in $s$ on each side of equation (28), we find

$$
s \tau_{N} \mathscr{E}(z) T(z) F(z)=\left(j / j_{0}\right) T(z),
$$

and the solution is

$$
F(z)=\frac{j}{j_{0}} \frac{1}{s \tau_{N} \mathscr{E}(z)} .
$$

The impedance corresponding to this solution is determined by equation (26),

$$
Z(s)-L / \epsilon s,
$$

which is the capacitive response expected at high frequencies for a structure with dielectric constant $\epsilon$ and length $L$.

At somewhat lower frequencies, when $s \tau_{N} \mathscr{E}$ is comparable to unity, we must also keep the linear

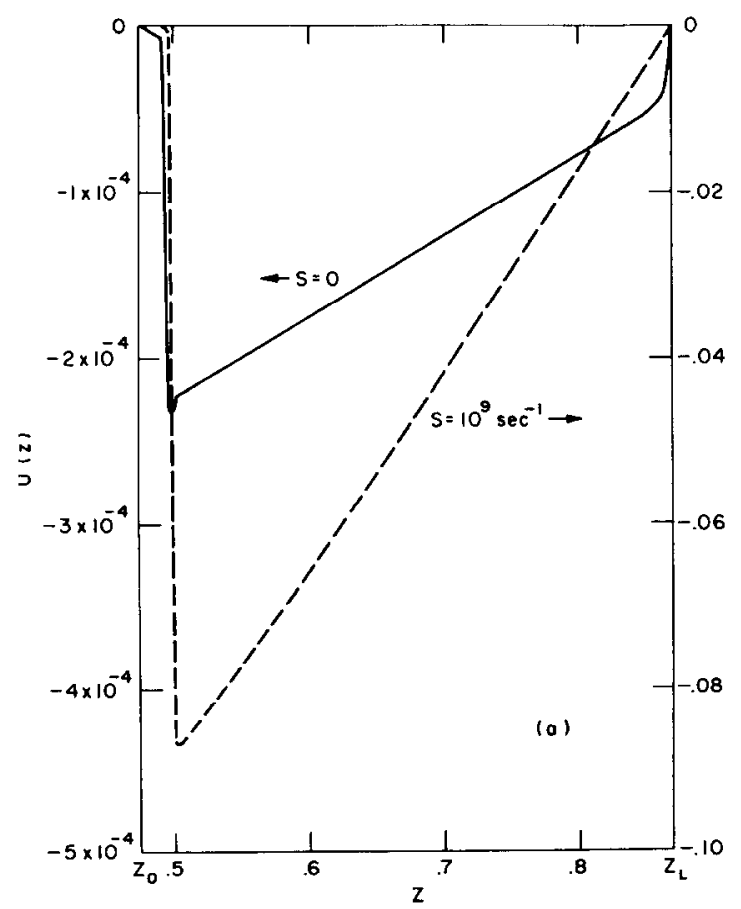

Fig. 1(a). $s$ term in $W(z)$. Since $T(z)$ is still large, however, we can still neglect $V(z)$ and $U(z)$. In this approximation equation $(28)$ becomes

$$
\left\{1+s \tau_{i} \mathscr{C}(z)\right\} T(z) F(z)=\left(j / j_{0}\right) T(z),
$$

and the solution is

$$
F(z)=\frac{j / j_{0}}{1+s \tau_{N} \mathscr{E}(z)}
$$

The integral (26) for the impedance cannot now be evaluated explicitly unless $\mathscr{E}(z)$ has some simple form. Some insight into the response at these frequencies can be gained, however, by writing $Z(s)$ as an integral over $x$. Using equations (26) and (37), we find

$$
Z(s)=\int_{0}^{L} \mathrm{~d} x \frac{E_{0}(x) / j_{0}}{1+s \in E_{0}(x) / j_{0}} .
$$

We can think of this integral as a series sum of

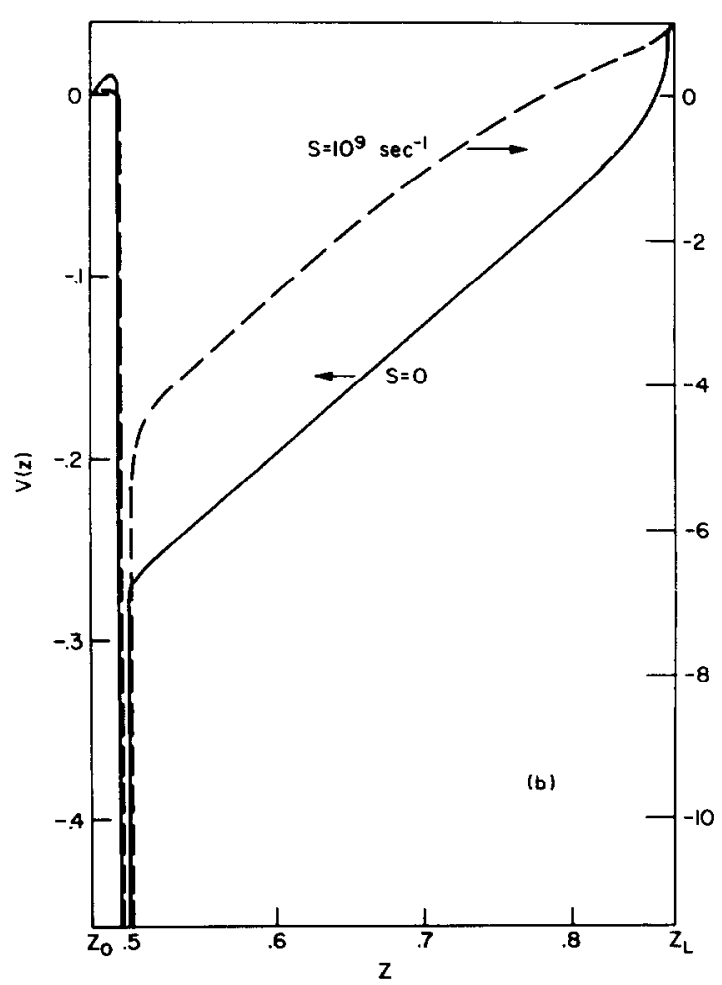

Fig. 1(b). 


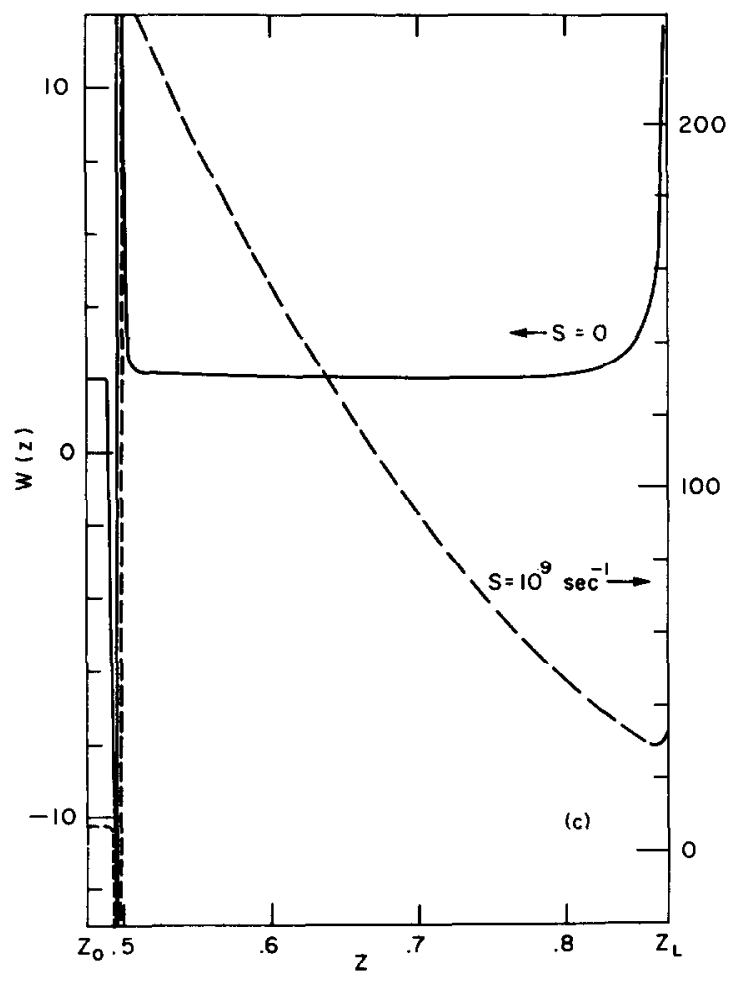

Fig. 1(c).

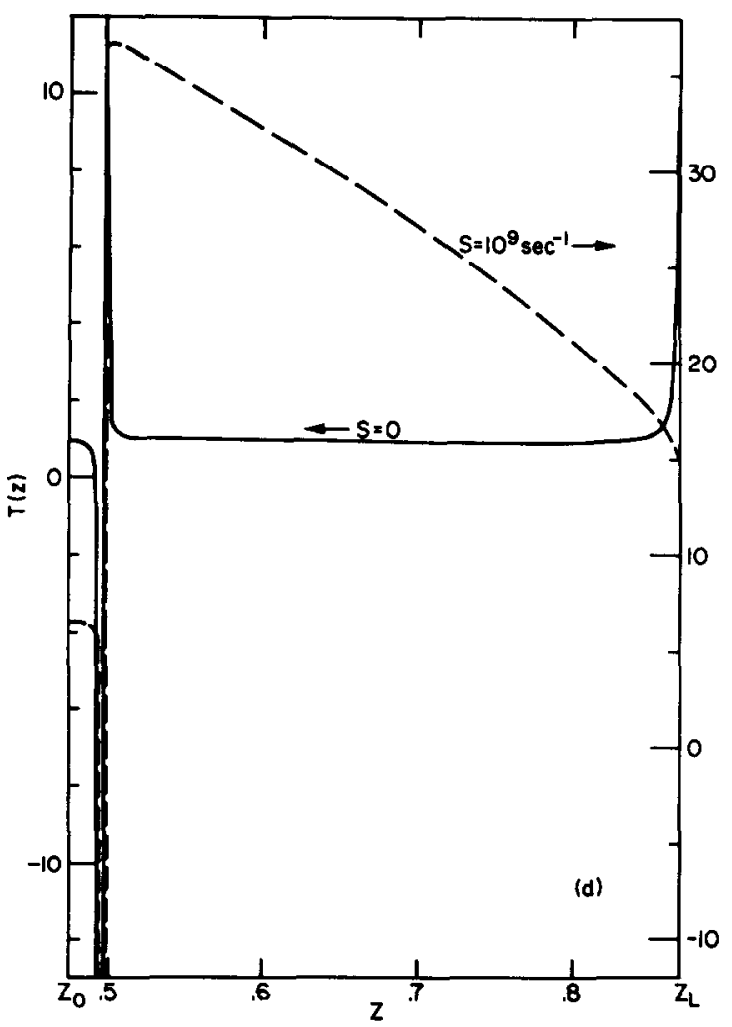

Fig. 1(d).

Fig. 1. Computed values of the coefficients in equation (28) for an Au-doped silicon $p-i-n$ device at $300^{\circ} \mathrm{K}$. The solid curves are for $s=0$, the dashed ones for $s=10^{9} \mathrm{sec}^{-1}$. The device parameters are $N_{R}=10^{16} \mathrm{~cm}^{-3}$,

$$
L=100 \mu \mathrm{m}, j_{0}=0.53 \mathrm{~A} / \mathrm{cm}^{2}, V_{0}=15.7 \mathrm{~V} \text {. }
$$

discrete elements each with impedance $\Delta Z$ given by

$$
\Delta Z=\frac{\Delta x E_{0}(x) / j_{0}}{1+s \epsilon E_{0}(x) / j_{0}} .
$$

We recognize that the impedance (39) is just what one would expect at high frequencies from a narrow slice of the structure with width $\Delta x$, i.e., a resistance $R(x)=\Delta x E_{0}(x) / j_{0}$ shunted by a capacitance $C(x)=\epsilon / \Delta x$. Thus the impedance (38) can be thought of as a series sum of parallel $R C$ elements as indicated in Fig. 2. We call this response the distributed $R C$ response. It can be expected to occur at high frequencies in any structure that has a nonuniform resistivity over a reasonable length.

\subsection{Small s behavior}

The device impedance in the low frequency limit

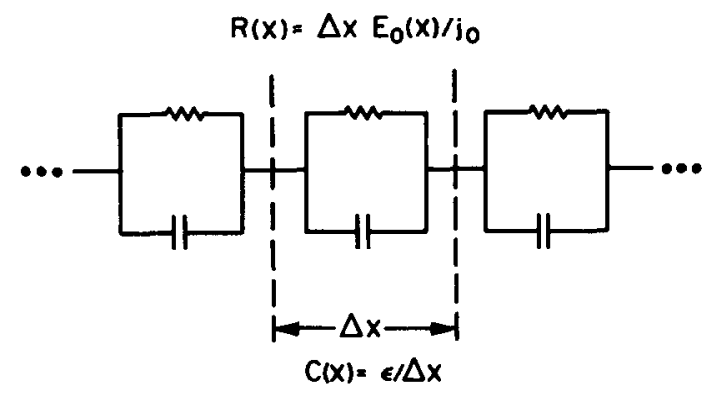

Fig. 2. Equivalent circuit for the distributed $R C$ response described in the text.

is determined simply by the slope of the $j_{0}-V_{0}$ curve, i.e.,

$$
Z(s \rightarrow 0)=\frac{\mathrm{d} V_{0}}{\mathrm{~d} j_{0}}
$$


a result which is obvious from physical considerations. To see how this result follows from the formal solution of equation (28) is more difficult. We will give a discussion only for the two squarelaw and the Lampert cube-law regimes of the current-voltage characteristic.

In the limit $s \rightarrow 0$ the expansion parameters $a$ and $b$ can be written as partial derivatives of the d.c. space charge, while $\alpha$ and $\beta$ can be written as partial derivatives of the d.c. recombination rate:

$$
\begin{aligned}
a \rightarrow \frac{\partial}{\partial n_{0}}\left(n_{0}-p_{01}+\right. & \left.N_{0}-P_{0}\right), \\
b & \rightarrow \frac{\partial}{\partial p_{0}}\left(p_{0}-n_{0}+P_{0}-N_{0}\right),
\end{aligned}
$$

$\alpha \rightarrow \frac{1}{N_{k}\left(c_{n}+c_{p}\right)} \frac{\partial r_{0}}{\partial n_{0}}, \quad \beta \rightarrow \frac{1}{N_{k}\left(c_{n}+c_{n}\right)} \frac{\partial r_{0}}{\partial p_{0}}$.

Through the relations (6), these derivatives can be changed to derivatives with respect to $z$ and $\mathscr{E}$. From the steady state results we know that in the square-law regimes the solution is given quite accurately by the quasineutrality approximation, and the narrow regions of large space charge can be neglected. Within this approximation $\mathrm{d} \mathscr{E} / \mathrm{d} z$ is constant, and $B(z)$ is proportional to $\mathscr{E}(z)$ over most of the device. This means that the expressions (41) can be evaluated explicitly. Using these results, it can be shown that the second term on the right side of equation (32) is negligible, and the first term gives

$$
T(z) \cong 1
$$

At the same time all but the first and third terms on the right side of equation (31) are negligible, which gives

$$
W(z) \cong 1+T(z) \cong 2 .
$$

Note that the exact results shown in the solid curves of Figs. 1(c) and 1(d) agree quite well with equations (42) and (43), if the space-charge regions are ignored.

Now we again make use of the fact that $U$ and $V$ are very small compared with $T$ and $W$, obtaining an approximate solution to equation (28) of the following form:

$$
F(z)=\frac{j}{j_{11}} \frac{T(z)}{W(z)} \cong \frac{1}{2} \frac{j}{j_{11}}
$$

Since the solution (44) gives $\mathrm{d} F / \mathrm{d} z=0$, it will be a valid approximation even if $U$ or $V$ are large, provided $T / W$ is slowly varying. The impedance can be easily evaluated by inspection of equation (27), yielding the required result for a square-law characteristic:

$$
Z(s \rightarrow 0)=\frac{1}{2} R_{11}
$$

The results (42)-(45) are equally valid for both the square-law regimes, although the derivation is simpler in the high injection case.

In the cube-law regime the only modification to the above results is that the second term on the right side of (31) also becomes important. In fact, this term becomes equal to the first and third terms, giving

$$
W(z)=3
$$

The impedance is thus

$$
Z(s \rightarrow 0)=\frac{1}{3} R_{0} .
$$

A solution with $F(z)$ constant implies that the small signal field has the same spatial dependence as the d.c. field, which in turn implies that the spatial dependence of the d.c. field does not change strongly with the applied voltage. We know from the computer solutions of the steady-state problem that in the square-law regimes the space-charge regions do move as the voltage is changed. Thus, we should expect the exact solutions for $F(z)$ to differ appreciably from the above approximate ones in these regions. However, since these regions are very narrow in the square-law regimes, the impedance will be only slightly affected by relatively large changes in $F(z)$ which might occur there.

\subsection{Low injection square-law approximations}

Since the oscillatory instabilities occur in the low injection square-law, then this is naturally the most interesting steady-state regime to discuss. Unfortunately, it is also the most difficult to discuss. The approximate d.c. solution in this regime is based on the quasineutrality approximation and the assumption of position- and injection-level- 
independent lifetimes. The $j_{0}-V_{0}$ curve is given by

$$
j_{10}=\frac{9}{8} e \mu_{n} \mu_{p}\left(p_{0} \tau_{n}-n_{0} \tau_{p}\right) \frac{V_{0}^{2}}{L^{3}},
$$

where $\tau_{n}$ and $\tau_{\nu}$ are the electron and hole lifetimes and $\left(p_{0} \tau_{n}-n_{0} \tau_{p}\right)$ is given by the capture and thermal emission coefficients of the traps. The corresponding approximate expressions for $\mathscr{E}$ and $B$ are

$$
\begin{gathered}
\mathscr{E}(z)=\frac{\left(\mu_{n} \tau_{n}+\mu_{p} \tau_{p}\right) N_{R}}{2\left(\mu_{n}+\mu_{p}\right)\left(p_{0} \tau_{n}-n_{0} \tau_{p}\right)}\left(z_{L}-z\right) \\
B(z)=\frac{\mu_{n}+\mu_{p}}{2 \mu_{n} \mu_{i}}\left(c_{n}+c_{p}\right) N_{R}\left(\mu_{n} \tau_{n}+\mu_{p} \tau_{p}\right) \mathscr{E}(z)
\end{gathered}
$$

and the corresponding small-signal parameters become

$$
\begin{gathered}
a=1+1 / s \tau_{n}, \quad b=1+1 / s \tau_{p}, \\
\alpha=\frac{s+1 / \tau_{n}}{\left(c_{n}+c_{p}\right) N_{R}}, \quad \beta=\frac{s+1 / \tau_{p}}{\left(c_{n}+c_{p}\right) N_{R}},
\end{gathered}
$$

where we have restricted $s$ to values such that $|s|$ is greater than the free carrier density multiplied by a typical capture coefficient. This restriction greatly simplifies the $s$ dependence in the problem, and the only error it introduces is in the low frequency limit which has already been discussed. Note that the electric field (49) fails to match the boundary condition at $z_{0}$. This is characteristic of the quasineutrality solutions.

Using the above results we find that equations (19)-(32) become

$$
\begin{gathered}
U(z)=-\frac{2 \mu_{n} \mu_{p} \tau_{n} \tau_{p}}{\left(\mu_{n} \tau_{n}+\mu_{p} \tau_{p}\right)^{2}} \frac{s \tau_{\epsilon}}{1+s \tau_{1}}\left(z_{L}-z\right), \\
V(z)=z-\frac{\mu_{n} \tau_{n}\left(1+s \tau_{p}\right)-\mu_{p} \tau_{p}\left(1+s \tau_{n}\right)}{\left(\mu_{n} \tau_{n}+\mu_{p} \tau_{p}\right)\left(1+s \tau_{t}\right)} \\
\times\left\{1+s \tau_{\epsilon}\left(z_{L}-z\right)\right\}, \\
W(z)=1+\left\{1+\frac{1}{2} s \tau_{\epsilon}\left(z_{L}-z\right)\right\} T, \\
T(z)=\frac{\left(1+s \tau_{n}\right)\left(1+s \tau_{p}\right)}{\left(1+s \tau_{1}\right)}
\end{gathered}
$$

where

$$
\tau_{1}=\frac{\tau_{n} \tau_{p}\left(\mu_{n}+\mu_{p}\right)}{\mu_{n} \tau_{n}+\mu_{p} \tau_{p}}, \quad \tau_{\epsilon}=\frac{\epsilon\left(\mu_{n} \tau_{n}+\mu_{p} \tau_{p}\right)}{e \mu_{n} \mu_{p}\left(p_{0} \tau_{n}-n_{0} \tau_{p}\right)} .
$$

Now solutions to equation (28) can be readily generated in terms of power series expansions about $z_{L L}$. This can be verified by inspection of the indicial equation. There is one serious difficulty, however. Since $U(z)$ no longer vanishes at $z_{0}$, this point is no longer a singular point of the differential equation. The two linearly independent power series solutions - one a particular, the other a homogeneous one-are both regular in the interval $z_{0} \leqslant z \leqslant z_{l}$. The complete solution is only defined with respect to an arbitrary constant multiplying the homogeneous solution, and in order to determine this constant we must impose a boundary condition.

In a previous paper[23] we discussed a simpler model which was equivalent to neglecting the $z$ dependence in all the quantities in equations (53)-(56). This model resulted in a second order differential equation for $F(z)$ with constant coefficients, and an explicit solution in terms of exponentials was obtained. Since that equation had no singular points, two boundary conditions were needed to determine the solution, and the ones we used were $F\left(z_{0}\right)=F\left(z_{t}\right)=0$. The corresponding boundary condition for the power series approach is $F\left(z_{0}\right)=0$. With these conditions the two models give very similar results.* Oscillatory instabilities are predicted, arising from poles in the impedance function, whose threshold conditions were shown to be in agreement with experiment. Because of the similarity between the results of the two approaches, we will omit a discussion of the power series method. The agreement with experiment and the agreement with our numerical results in Section 4 are the primary justifications for the apparently arbitrary choice of boundary conditions.

There is one important feature of the impedance function which can be demonstrated independent of the boundary conditions. Namely,

$$
Z(s)=0 \text { at } s=-1 / \tau_{n},-1 / \tau_{p} .
$$

This result follows from equation (56). When $T(z)=0$, then $F$ and also $Z$ must vanish. These zeros of $Z(s)$ represent the decaying modes observed in a transient response experiment under constant voltage conditions [22].

\footnotetext{
${ }^{*}$ Curve 1 in Fig. 1 of Ref. [23] is the result of the above power series solution.
} 
The $s \rightarrow 0$ limit of equations (53)-(56) seems to give the correct low frequency result: $T=1$, $W=2$, and thus $F(z)=\frac{1}{2}\left(j / j_{0}\right)$. However, this result is not obtained as a limit of the solutions for nonzero $s$ from either of the models described above. In fact, the solutions from both models yield impedances which oscillate rapidly without converging in the limit $s \rightarrow 0$. These oscillations are not physical. They stem from the fact that $U(z)$ in equation (53) tends to zero as $s$ does. The difficulty arises from the approximate expressions (51) used for $a$ and $b$. These expressions diverge as $s \rightarrow 0$, whereas the actual values remain finite.

\section{NUMERICAL SOLUTION AND DISCUSSION}

In this Section we discuss two different methods for finding a numerical solution to equation (28), only one of which was successful. The primary motivation for obtaining such a solution is to confirm the results of our simple model which was successful in explaining the experimental results. In principle at least this should be a relatively simple task. The coefficients $U, V, W$, and $T$ are all continuous, bounded functions of $z$. The solution $F(z)$, which satisfies the boundary conditions of regularity at the endpoints, exists and is unique.

The method which we tried first was to treat (28) as a pair of first order equations of the form:

$$
\begin{gathered}
\frac{\mathrm{d} F}{\mathrm{~d} z}=G(z) \\
\frac{\mathrm{d} G}{\mathrm{~d} z}=\left[\left(j / j_{0}\right) T(z)-V(z) G(z)\right. \\
-W(z) F(z)] / U(z) .
\end{gathered}
$$

These equations were integrated simultaneously starting out at the boundaries and using one of the standard techniques based on numerical integration [35]. The solution obtained starting from the left boundary contains an arbitrary constant multiplying the homogeneous solution regular at $z_{0}$. Similarly, the solution obtained starting from the right boundary contains another arbitrary constant multiplying the homogeneous solution regular at $z_{l}$. These two constants are determined by matching the right and left hand solutions and their first derivatives at some intermediate point $z_{i}$.
By constructing $F(z)$ and then the impedance function in the above manner, one can show that $Z(s)$ will have poles whenever the Wronskian of the two homogeneous solutions vanishes:

$$
\left(F_{h}{ }^{0} \frac{\mathrm{d}}{\mathrm{d} z} F_{h}{ }^{L}-F_{h}{ }^{L} \frac{\mathrm{d}}{\mathrm{d} z} F_{h}{ }^{0}\right)_{z=z_{i}}=0
$$

where the superscripts indicate the boundary at which each function is regular. The condition (59), which is independent of $z_{i}$, will presumably be satisfied at some discrete values of $s$. If these values occur in the right-half $s$ plane, spontaneous oscillations will occur.

Although the above method is very straightforward and leads to a simple condition for oscillations, in practice it did not work. The difficulty arose in integrating (58) across the space-charge regions. Referring back to Fig. 1 we see that the coefficients in equation (28), although continuous, have very sharp structure in these regions. We tried a variety of numerical integration techniques, but none succeeded in taking the solutions smoothly through the spacecharge regions. With unlimited computer time this method will surely work. With a given amount of computer time, however, we had much better success with an entirely different approach.

We begin by assuming an expansion for $F(z)$ of the form:

$$
F(z)=\sum_{i=1}^{\prime} f_{i} P_{i}(z)
$$

where $\left\{P_{i}(z)\right\}$ is any set of complete functions regular at the boundaries. For our problem we chose the Legendre polynomials suitably normalized over the interval $z_{0} \leqslant z \leqslant z_{L}$. We next insert (60) into (28), multiply on the left by $P_{k}(z)$. and integrate. The result is a set of $I$ simultancous linear equations for $f_{i}$ :

$$
\sum_{i=1}^{l} S_{k i} f_{i}=T_{k}, \quad(k=1,2, \ldots, I),
$$

where

$$
\begin{gathered}
S_{k i}=\int_{z,}^{z_{L}} \mathrm{~d} z P_{k}(z)\left\{U \frac{\mathrm{d}^{2}}{\mathrm{~d} z^{2}}+V \frac{\mathrm{d}}{\mathrm{d} z}+W\right\} P_{i}(z) \\
T_{k}=\left(j / j_{0}\right) \int_{z \|}^{z_{L}} \mathrm{~d} z T(z) P_{k}(z)
\end{gathered}
$$


The set of equations (61) is then solved for $f_{i}$, and the resulting impedance function is

$$
Z(s)=R_{0} \sum_{i=1}^{I} g_{i} f_{i},
$$

where

$$
g_{i}=\frac{\int_{z_{0}}^{z_{L}} \mathrm{~d} z \mathscr{E}(z) B(z) P_{i}(z)}{\int_{z_{0}}^{z_{L}} \mathrm{~d} z \mathscr{E}(z) B(z)} .
$$

The above method will always converge to the correct solution in the limit $I \rightarrow \infty$. In practice, of course, we must truncate the expansion at some reasonable value of $I$ and hope the result is still valid. The truncated solutions will not agree with the actual solutions in the narrow space-charge regions. This is expected since the sharp structure in these regions (of the sort shown in Fig. 1) could only be fit with very high order polynomials. To the extent that these regions do not strongly influence the device impedance, then the truncated solutions will be quite accurate. Here we see the real advantage of the polynomial expansion method - it tends to smooth out the effects of the space-charge regions. The method based on integrating the differential equation from the boundaries must find the solution in these regions just as accurately as in the rest of the device, since the errors will propagate.

To test the accuracy of our expansion method, we first applied it to the case $s=0$. The solid curve in Fig. 3 is the $s=0$ solution $F(z)$ obtained for the same steady-state conditions as in Fig. 1, using ten Legendre polynomials in the expansion. The dashed curve is a plot of $T(z) / W(z)$ in the regions where the ratio is slowly varying. From equation (44) we expect the dashed curve to match the correct solution except in the space-charge regions. Although $F(z)$ has oscillations induced by the attempt to fit sharp structure in these regions, over most of the device its average value is given fairly well by the dashed curve. Moreover, the impedance given by the solution in Fig. 3 is within $4 \%$ of the exact value $0.489 R_{0}$ determined by the slope of the $j_{0}-V_{0}$ curve. As a further check on the convergence of this method, we computed the impedance using six Legendre polynomials and found the result to be within $6 \%$ of the exact value.

In Fig. 4 we show the computed real and imaginary parts of the impedance along the

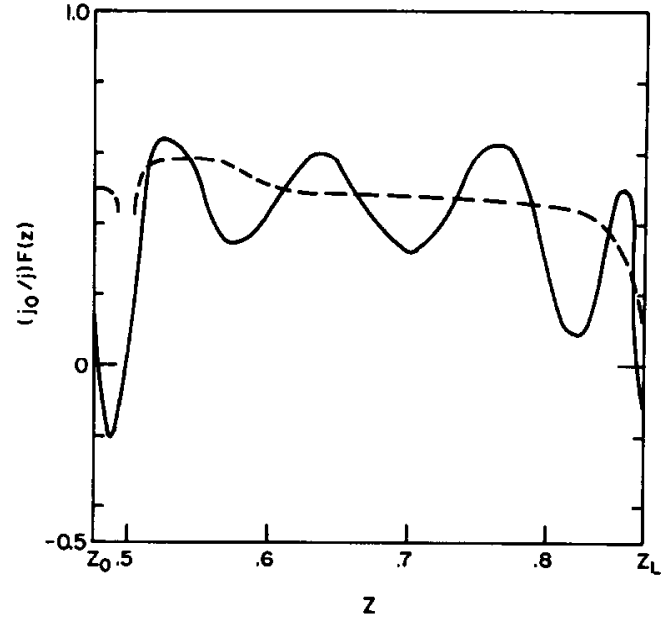

Fig. 3. Computer solution $F(z)$ of equation (28) at $s=0$ using the same parameters as those for Fig. 1. The dashed curve is a plot of $T / W$ in the regions where it is slowly varying. The impedance from this solution is $0.47 R_{0}$, compared with the exact value of $0.489 R_{0}$.

imaginary $s$ axis $(s=i \omega)$. Ten Legendre polynomials are used in this calculation, and the steadystate parameters are $N_{R}=10^{16} \mathrm{~cm}^{-3}, L=100 \mu \mathrm{m}$, $j_{0}=4.71 \mathrm{~A} / \mathrm{cm}^{2}$, and $V_{0}=45.09 \mathrm{~V}$. Also shown in the figure as dashed curves are the corresponding results from the simple model of Ref.[23], where we used $\tau_{n}=18.2 \mathrm{nsec}$ and $\tau_{p}=3.53 \mathrm{nsec}$, the low injection square-law lifetimes. Resonant structure occurs at the same frequencies in both calculations, and for large $\omega$ both match smoothly onto the high frequency limit (35). The results from the two models are quantitatively very similar. The main difference being that, for the particular operating point chosen, the pole causing the high frequency resonance is nearer the $\omega$ axis in the exact solution. The computational difficulties are quite different, however. For example, the solid curves in Fig. 4 required approximately $20 \mathrm{~min}$ of machine time on the Honeywell 6000 digital computer, while the dashed curves required only $20 \mathrm{sec}$.

The accuracy of our impedance in the limits of low and high frequencies, where it can be easily checked, was found to be several percent. Since the numerical difficulties are no worse at intermediate values of $s$, we expect the same magnitude of accuracy to hold there as well.

We have not attempted to make a detailed comparison of our computer solution with experimental results. Such a comparison seems to be 


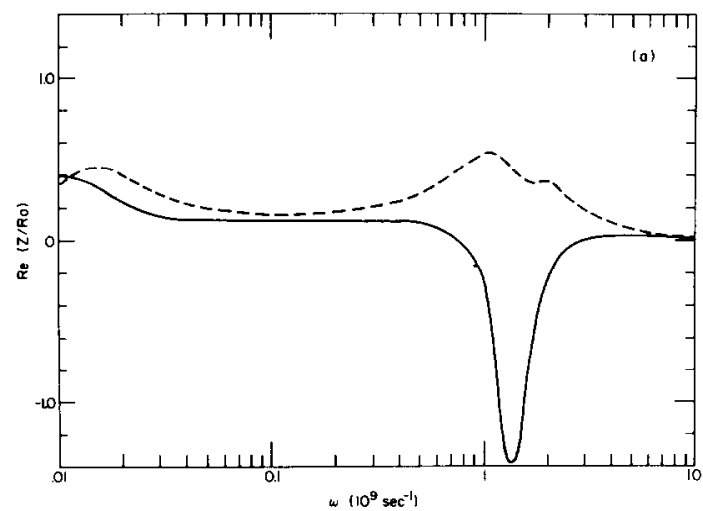

Fig. 4(a).

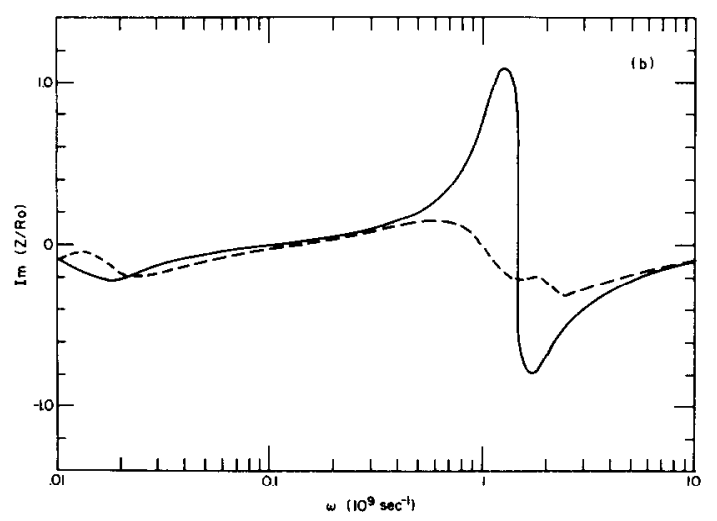

Fig. 4(b).

Fig. 4. Computed real (a) and imaginary(b) parts of the impedance on the imaginary $s$ axis $(s=i \omega)$ for an Audoped silicon $p-i-n$ device at $300^{\circ} \mathrm{K}$. The solid curve is the result of solving the exact equation (28) using the polynomial expansion method with ten Legendre polynomials. The dashed curve is the result from equation (14) in Ref. [23]. The device parameters are $N_{R}=10^{16}$ $\mathrm{cm}^{-3}, L=100 \mu \mathrm{m}, j_{0}=4.71 \mathrm{~A} / \mathrm{cm}^{2}, V_{0}=45.09 \mathrm{~V}$. The low injection square-law lifetimes, $\tau_{n}=18.2 \mathrm{nsec}$ and $\tau_{p}=$ $3.53 \mathrm{nsec}$, were used for the dashed curve.

unwarranted for several reasons: First, it would require an inordinate amount of computer time. Second, the present solution gives results which tend to confirm our simple model[23], and that model was shown to agree with experiment. Third, the main differences between the two models will arise from the narrow space-charge regions near the contacts, which are completely neglected in the simple model. In an actual device diffusion will be important in these regions and will tend to modify the boundary conditions. Both models neglect diffusion, however, so that any differences caused by including the space-charge regions will have questionable validity with regard to an actual device.

However, the problem of finding an efficient numerical method for accurately solving equation (28) still is of considerable mathematical interest. The difficulty is with the sharp structure in the narrow space-charge region, and if techniques were developed to handle this problem one would expect them to have broad applicability.

One very interesting result that came out of our numerical solutions was that the small-signal quasineutrality solution[22] does not give the correct impedance throughout the expected range in $s$. The quasineutrality solution, which neglects all space-charge effects, is given by

$$
Z(s)=R_{0} T /(1+T),
$$

where $T$ is given by (56). This equation gives the correct zeros of $Z$ on the real $s$ axis, and normally one assumes it will be valid whenever $|s| \ll \tau_{t l}{ }^{-1}$. where $\tau_{d}$ is the local dielectric relaxation time. However, for the data in Fig. 4 at $\omega=5 \times 10^{7} \mathrm{scc}^{-1}$, a factor of 20 less than $\tau_{d}^{-1}$, the real part of $Z$ from equation (66) is several times greater than the correct value. The reason for the large discrepancy is that small changes in the free carrier densities can lead, through trapping, to much larger changes in the trapped densities and, hence, in the space charge associated with the traps.

The failure of quasineutrality actually provides the key to understanding the oscillatory instabilities. A small wavelike fluctuation in the free carrier densities leads to a larger fluctuation in the trapped densities, shifted by $90^{\circ}$. The trapped space charge leads to a field further shifted by $90^{\circ}$, resulting in a $180^{\circ}$ shift of the field from the free carriers. If the magnitude of the resulting field is such that the various contributions to the small-signal current cancel, then the system will oscillate freely in that mode.

We show in Fig. 5 the field and charge number densities as a function of position for the oscillatory mode at threshold, i.e., when the pole giving the oscillations crosses the $\omega$ axis. Figure $5(\mathrm{~b})$ is the configuration $1 / 4$ cycle in time later than $5(\mathrm{a})$. These curves were calculated from the power series method of Section 3.4 using the approximate 


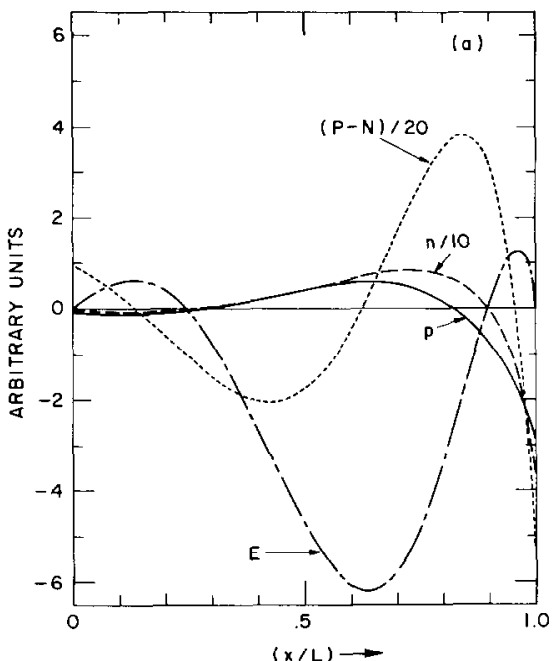

Fig. 5(a).

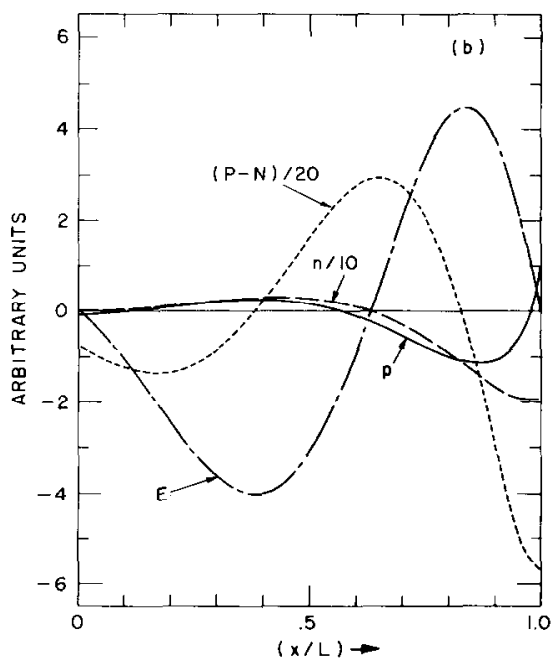

Fig. 5(b).

Fig. 5. The time dependent electric field and charge number densities as a function of position at the threshold for space-charge recombination oscillations in a $p-i-n$ device. The curves were calculated using the power series method of Section 3.4 with the boundary condition $F\left(z_{0}\right)=0$. The parameters chosen were $\omega=2.12 \times 10^{7}$ $\mathrm{sec}^{-1},\left(V_{0} / L^{2}\right)=6.42 \times 10^{5} \mathrm{~V} / \mathrm{cm}^{2},\left(j_{0} / L\right)=2.68 \times 10^{3}$ $\mathrm{A} / \mathrm{cm}^{3}, \tau_{n}=36.4 \mathrm{nsec}, \tau_{p}=1.765 \mathrm{nsec}$, and the mobilities were those for silicon at $300^{\circ} \mathrm{K}$. (b) is the configuration $\frac{1}{4}$ cycle in time later than (a).

equations (53)-(56) and the boundary condition $F\left(z_{0}\right)=0$. The phase relationships described above are evident in the figure. Here we have chosen $\tau_{n} \gg \tau_{\mu}$ which implies that $n_{0} E$ and $E_{0} n$ are the dominant contributions to the small-signal current. As shown in the figure $n$ and $E$ remain nearly $180^{\circ}$ out of phase across the device, resulting in $n_{0} E+E_{0} n \cong 0$.

Also indicated in Fig. 5 is the fact that the oscillation takes the form of an asymmetrical left-running wave. The asymmetry comes from the $z$ dependence in $U, V$, and $W$ included in the power series method. A similar picture of leftrunning waves resulted from our simple model of Ref.[23], but there the waves were more symmetrical since a spatially homogeneous d.c. solution was assumed.

\section{REFERENCES}

1. N. Ilolonyak, Jr, and S. F. Bevacqua, Appl. Phys. Lett. 2, 71 (1963).

2. J. S. Moore, N. Holonyak, Jr., and M. D. Sirkis, Solid-St.Electron. 10, 823 (1967).

3. M. M. Blouke, N. Holonyak, Jr., B. G. Streetman and H. R. Zwicker, Solid-St. Electron. 13, 337 (1970).

4. V.P. Sondaevskii and V. I. Stafeev, Fiz. Tverd. Tela 6, 80 (1964). [Soviet Phys. Solid St. 6, 63 (1964)].

5. I. V. Karpova and S. G. Kalashnikov, $Z$ h. Eksperm. $i$ Teor. Fiz. Fis'ma v Redaktsiyu 6, 954 (1967). [Soviet Phys.JETP Lett. 6, 369 (1967)].

6. F. M. Berkovskii and R. S. Kasymova, Fiz. Tverd. Tela 8, 1985 (1966). [Soviet Phys. Solid St. 8, 1580 (1967)].

7. W. Viehmann, Appl. Phys. Lett. 14, 39 (1969.

8. B. G. Streetman, N. Holonyak, Jr., H. V. Krone, W. Dale Compton, Appl. Phys. Lett. 14, 63 (1969).

9. C. C. Lin, The Theory of Hydrodynamic Stability, Cambridge University Press, Cambridge (1966).

10. O. V. Konstantinov and V. I. Perel, Fiz. Tverd. Tela 6, 3364 (1964). [Soviet Phys. Solid St. 6, 2691 (1965)].

11. O. V. Konstantinov, V. I. Perel and G. V. Tsarenkov, Fiz. Tverd. Tela 9, 1761 (1967) [Soviet Phys. Solid St. 9, 1381 (1967)].

12. P. Antognetti, A. Chiabrera and S. Ridella, Appl. Phys. Lett. 18, 544 (1971).

13. P. Antognetti, A. Chiabrera and S. Ridella, Solid-St. Electron. 14, 1123 (1971).

14. J. S. Moore, Ph.D. Thesis, University of Illinois (1967).

15. M. Brousseau, J. Barrau, J. C. Brabant and Nguyen van Tuyen, Solid-St. Electron. 13,906 (1970).

16. R. Baron, O. J. Marsh and J. W. Mayer, J. appl. Phys. 37, 2614 (1966).

17. H. R. Bilger, D. H. Lee, M. A. Nicolet and E. R. McCarter, J. appl. Phys. 39, 5913 (1968).

18. F. Driedonks, Physica 46, 291 (1970).

19. A. van der Ziel, Solid-St. Electron. 13, 191 (1970).

20. A. van der Ziel, Solid-St. Electron. 13, 195 (1970).

21. R. Baron and J. W. Mayer, in Semiconductors and Semimetals (edited by R. K. Willardson and A. C. Beer), Vol. 6, Academic Press, New York (1970). 
22. W. H. Weber, R. S. Elliott and A. L. Cederquist, J. appl. Phys. 42, 2497 (1971).

23. W. H. Weber and G. W. Ford, Appl. Phys. Lett. 18 , 241 (1971).

24. M. A. Lampert and A. Rose, Phys. Rev. 121, 26 (1961).

25. M. A. Lampert, Phys. Rev. 125, 126 (1962).

26. K. L. Ashley, Ph.D. Thesis, Carnegie Institute of Technology (1963).

27. K. L. Ashley and A. G. Milnes, J. appl. Phys. 35, 369 (1964).

28. Willes H. Weber and G. W. Ford, Solid-St. Electron. 13,1333 (1970)

29. H. J. Deuling, J. appl. Phys. 41, 2179 (1970).

30. H. R. Zwicker, B. G. Streetmen, A. M. Andrews and H. J. Deuling, Appl. Phys. Lett. 16, 63 (1970).

31. M. A. Lampert and P. Mark, Current Injection in Solids, Academic Press, New York (1970).

32. W. Shockley and W. T. Read, Jr., Phys. Rev. 87, 835 (1952).

33. W. Shockley, Proc. Inst. Radio Engrs 46, 973 (1958).

34. E. T. Whittaker and G. N. Watson, Modern Analysis, 4th Ed. Chapter X, p. 194, Cambridge University Press, Cambridge (1952).

35. P. Henrici, Discrete Variable Methods in Ordinary Differential Equations, Chapter 5, Wiley, New York (1962).

36. J. M. Fairfield and B. V. Gokhale, Solid-St. Electron. 8,685 (1965).
The solution of the small-signal analogues of equations (A1) yields the following expressions for $a$ and $b$ :

$$
\begin{aligned}
a= & 1+D\left\{c_{n}\left(N_{R}-N_{0}-P_{0}\right)\left(s+2 e_{n}+2 c_{p} p_{0}+e_{p}{ }^{+}+c_{n}{ }^{+} n_{0}\right)\right. \\
& \left.+c_{n}{ }^{+} P_{0}\left(s+2 e_{p}+2 c_{n} n_{0}+e_{n}{ }^{-}+c_{p}{ }^{-} p_{0}\right)\right\},
\end{aligned}
$$

$$
b=1+D\left\{c_{p}\left(N_{R}-N_{0}-P_{0}\right)\left(s+2 e_{p}+2 c_{n} n_{0}+e_{n}{ }^{-}+c_{p}{ }^{-} p_{0}\right)\right.
$$$$
\left.+c_{p}{ }^{-} N_{0}\left(s+2 e_{n}+2 c_{p} p_{0}+e_{p}{ }^{+}+c_{n}{ }^{+} n_{0}\right)\right\} \text {, }
$$

where

$$
\begin{aligned}
D^{-1}= & \left(s+e_{n}{ }^{-}+c_{p}{ }^{-} p_{0}\right)\left(s+e_{p}{ }^{+}+c_{n}{ }^{+} n_{0}\right) \\
& +\left(s+e_{n}{ }^{-}+c_{p}{ }^{-} p_{0}\right)\left(e_{n}+c_{p} p_{0}\right) \\
& +\left(s+e_{p}{ }^{+}+c_{n}{ }^{+} n_{0}\right)\left(e_{p}+c_{n} n_{0}\right) .
\end{aligned}
$$

If $|s|$ is greater than the thermal emission coefficients and the products of the capture cocfficients with the carrier densities, then $D \cong s^{-2}$, and we recover the result (51), i.e., $a \rightarrow 1+1 / s \tau_{n}$ and $b \rightarrow 1+1 / s \tau_{p}$.

The d.c. recombination rates are given by equations (2.8) in Ref. [28]:

$$
\begin{aligned}
& r_{n, 0}=\left(c_{n} n_{0}-e_{n}\right)\left(N_{A}-N_{0}-P_{0}\right)-e_{n}{ }^{-} N_{0}+c_{n}{ }^{+} n_{0} P_{0}, \\
& r_{p, 0}=\left(c_{p} p_{0}-e_{p}\right)\left(N_{R}-N_{0}-P_{0}\right)-e_{p}{ }^{+} P_{0}+c_{p}{ }^{-} p_{0} N_{0} .
\end{aligned}
$$

Using (A2) one can show that $r_{n, 0}=r_{p, 0}=r_{0}$, where

$$
r_{0}=N_{R}\left(n_{0} p_{0}-n_{i}^{2}\right) \frac{c_{n}{ }^{+} c_{p}\left(e_{n}{ }^{-}+c_{p}{ }^{-} p_{0}\right)+c_{n} c_{p}-\left(e_{p}++c_{n}{ }^{+} n_{0}\right)}{\left(e_{p}+c_{n} n_{0}\right)\left(e_{p}{ }^{+}+c_{n}{ }^{+} n_{0}\right)+\left(e_{n}+c_{p} p_{0}\right)\left(e_{n}{ }^{-}+c_{p}{ }^{-} p_{0}\right)+\left(e_{p}{ }^{+}+c_{n}{ }^{+} n_{0}\right)\left(e_{n}{ }^{-}+c_{p}{ }^{-} p_{0}\right)}
$$

\section{APPENDIX}

Here we give expressions for the quantities used in our numerical solutions for Au-doped silicon devices. The formulas will be given in terms of $n_{0}$ and $p_{0}$. It is assumed that a solution $\mathscr{E}(z)$ has been determined numerically, and thus using (6) all quantities can be determined as functions of $z$ alone.

The rate equations for the trapped charge densities are given by equations (2.9) in Ref. [28]:

$\frac{\partial N_{0}}{\partial t}=\left(c_{n} n_{0}+e_{p}\right)\left(N_{R}-N_{0}-P_{0}\right)-\left(c_{p}{ }^{-} p_{0}+e_{n}{ }^{-}\right) N_{0},(\mathrm{Al})$

$\frac{\partial P_{0}}{\partial t}=\left(c_{p} p_{0}+e_{n}\right)\left(N_{R}-N_{0}-P_{0}\right)-\left(c_{n}{ }^{+} n_{0}+e_{p}{ }^{+}\right) P_{0}$

where $e_{p}\left(e_{n}\right)$ and $e_{p}{ }^{+}\left(e_{n}^{-}\right)$are the thermal emission coefficients for holes (electrons) from neutral and positive (negative) gold recombination centers, respectively; while $c_{p}\left(c_{n}\right)$ and $c_{p}{ }^{-}\left(c_{n}{ }^{+}\right)$are the corresponding capture coefficients. The steady-state solution of (A1) yields and $n_{i}$ is the free carrier density in intrinsic silicon.

The solution of the small-signal analogues of (A5) yield the following expressions for $\alpha$ and $\beta$ :

$$
\begin{aligned}
& \left(c_{n}+c_{p}\right) N_{k} \alpha=s+c_{n}\left(N_{k}-N_{0}-P_{0}\right)\left[1-D\left\{\left(s+e_{n}{ }^{+}\right.\right.\right. \\
& \left.+c_{n}{ }^{+} n_{0}\right)\left(c_{n} n_{0}-e_{p}+c_{p} p_{0}-e_{n}-c_{p} p_{0}+e_{n}{ }^{-}\right) \\
& \left.\left.+\left(e_{n}+c_{p} p_{0}\right)\left(c_{n} n^{\prime}-e_{p}{ }^{\prime}-c_{p} p_{0}+e_{n}\right)\right\}\right] \\
& +c_{n}{ }^{+} P_{0}\left[1+D\left\{( s + e _ { n } { } ^ { - } + c _ { p } { } ^ { - } p _ { 0 } ) \left(c_{n} n_{0}-e_{p}+c_{p} p_{0}-e_{n}\right.\right.\right. \\
& \left.\left.\left.-c_{n}{ }^{+} n_{0}+e_{n}{ }^{+}\right)+\left(e_{p}+c_{n} n_{0}\right)\left(c_{n}{ }^{-} p_{0}-e_{n}{ }^{-}-c_{n}{ }^{+} n_{0}+e{ }^{+}\right)\right\}\right], \\
& \left(c_{n}+c_{p}\right) N_{R} \beta=s+c_{n}\left(N_{R}-N_{t}-P_{0}\right)\left[1-D\left\{\left(s+e_{n}\right.\right.\right. \\
& \left.+c_{p}{ }^{-} p_{0}\right)\left(c_{n} n_{0}-e_{n}+c_{p} p_{0}-e_{n}-c_{n}{ }^{+} n_{00}+e_{p}{ }^{+}\right) \\
& \left.\left.+\left(e_{p}+c_{n} n_{0}\right)\left(c_{p}{ }^{-} p_{0}-e_{n}-c_{n}{ }^{+} n_{0}+e_{p}{ }^{+}\right)\right\}\right] \\
& +c_{p}{ }^{-} N_{0}\left[1+D\left\{( s + e _ { p } { } ^ { + } + c _ { n } { } ^ { + } n _ { 0 } ) \left(c_{n} n_{0}-e_{p}+c_{p} p_{0}-e_{n}\right.\right.\right. \\
& \left.\left.-c_{n}{ }^{-} p_{0}+e_{n}{ }^{-}\right)+\left(e_{n}+c_{n} p_{0}\right)\left(c_{n}{ }^{+} n_{0}-e_{n}{ }^{+}-c_{n}{ }^{-} p_{0}+c_{n}{ }^{-1}\right\}\right] \text {. }
\end{aligned}
$$

If $|s|$ is in the range such that equations (51) are valid, then the terms involving $D$ in (A7) can be neglected, and we obtain the results given in equations (52).

$$
\begin{aligned}
& N_{0}=N_{k} \frac{\left(e_{p}+c_{n} n_{0}\right)\left(e_{p}{ }^{+}+c_{n}{ }^{+} n_{0}\right)}{\left(e_{p}+c_{n} n_{0}\right)\left(e_{p}{ }^{+}+c_{n}{ }^{+} n_{0}\right)+\left(e_{n}+c_{p} p_{0}\right)\left(e_{n}{ }^{-}+c_{p}{ }^{-} p_{0}\right)+\left(e_{p}{ }^{+}+c_{n}{ }^{+} n_{0}\right)\left(e_{n}{ }^{-}+c_{p}{ }^{-} p_{0}\right)} \\
& P_{0}=N_{k} \frac{\left(e_{n}+c_{p} p_{0}\right)\left(e_{n}{ }^{-}+c_{p}{ }^{-} p_{0}\right)}{\left(e_{p}+c_{n} n_{0}\right)\left(e_{p}{ }^{+}+c_{n}{ }^{+} n_{0}\right)+\left(e_{n}+c_{p} p_{0}\right)\left(e_{n}{ }^{-}+c_{p}{ }^{-} p_{0}\right)+\left(e_{p}{ }^{+}+c_{n}{ }^{+} n_{0}\right)\left(e_{n}{ }^{-}+c_{p}, p_{0}\right)} .
\end{aligned}
$$

